archives-ouvertes

\title{
L'action collective dans les territoires. Questions structurantes et fronts de recherche
}

Laurence Amblard, Karim Berthome, Marie Houdart, Sylvie Lardon

\section{To cite this version:}

Laurence Amblard, Karim Berthome, Marie Houdart, Sylvie Lardon. L'action collective dans les territoires. Questions structurantes et fronts de recherche. Géographie, Économie, Société, Lavoisier, 2020, 10.3166/ges.20.2017.0032 . hal-02435040

\section{HAL Id: hal-02435040 \\ https://hal.archives-ouvertes.fr/hal-02435040}

Submitted on 10 Jan 2020

HAL is a multi-disciplinary open access archive for the deposit and dissemination of scientific research documents, whether they are published or not. The documents may come from teaching and research institutions in France or abroad, or from public or private research centers.
L'archive ouverte pluridisciplinaire HAL, est destinée au dépôt et à la diffusion de documents scientifiques de niveau recherche, publiés ou non, émanant des établissements d'enseignement et de recherche français ou étrangers, des laboratoires publics ou privés. 
L'action collective dans les territoires. Questions structurantes et fronts de recherche

Laurence Amblard, Guy-El-Karim Berthomé, Marie Houdart et Sylvie Lardon 


\section{Introduction}

De nombreux travaux empiriques et théoriques ont démontré que des acteurs sont capables, dans le cadre d'actions collectives décentralisées, de négocier et de respecter des accords permettant une gestion durable d'un bien commun (Ostrom, 1990 ; Dubuisson-Quellier, 2001; Hatchuel, 2001 ; Adger, 2003 ; Lindberg et Fahlbeck, 2011). A l'échelle des territoires, les travaux s'intéressant à l'action collective (Ac) ont permis la compréhension des jeux d'acteurs participant au développement des territoires, ainsi que l'identification de leviers d'action pour accompagner les mutations des territoires ou la gestion de ressources communes. Plus précisément, dans les domaines du développement des territoires ruraux et périurbains, de la gestion des ressources environnementales, de l'agriculture, de l'aménagement, de l'alimentation, les recherches sur l'Ac constituent un champ donnant lieu à de nombreuses analyses empiriques (Lubell et al., 2002 ; Meinzen-Dick et al., 2004 ; Goldman et al., 2007 ; German et Hailemichael, 2008 ; Markelova et al., 2009). La diversité, à la fois des travaux théoriques et empiriques, témoigne de la vitalité de ce champ de recherche.

Dans l'optique d'apporter une contribution à la capitalisation et à la clarification des travaux portant sur l'action collective en lien avec le territoire, cet article propose une synthèse des travaux en géographie et économie appliqués aux champs de l'aménagement, de la gestion des ressources environnementales et du développement des territoires ruraux et périurbains. Cette synthèse donne à voir les apports d'une entrée territoriale de l'action collective, en termes de production de connaissances scientifiques et de connaissances pour l'action. Elle repose sur une analyse, par les auteurs, des travaux menés par des chercheurs membres de l'UMR Territoires ${ }^{1}$ sur l'action collective dans les territoires. L'analyse a bénéficié d'une

\footnotetext{
${ }^{1}$ Née en 2017 de la fusion de l'UMR Métafort et du Ceramac, l'UMR Territoires regroupe une centaine de membres, sous les tutelles AgroparisTech, Inra, Irstea, Université Clermont-Auvergne et VetAgro Sup (http://www.metafort.fr et http://ceramac.univ-bpclermont.fr)
} 
réflexion collective associant les chercheurs de l'unité dans le cadre de deux séminaires. Elle est mise en regard d'une revue de la littérature nationale et internationale. L'analyse de ce corpus nous a amenés à identifier trois questionnements structurant les apports des travaux sur l'action collective en lien avec le territoire (Fig. 1) :

(1) Dans quelle mesure les territoires conditionnent-ils le développement des actions collectives? Quelle est la part des facteurs territoriaux et supra-territoriaux dans le développement de l'action collective?

(2) Les actions collectives prennent différentes formes dans les territoires. En quoi ces formes sont-elles liées aux spécificités des territoires ? Comment se constitue une base d'adhésion commune parmi les acteurs concernés ? Quelle est la contribution des différents acteurs à la dynamique collective?

(3) Enfin, en retour, comment l'action collective contribue-t-elle au développement territorial, génère-t-elle de nouvelles ressources et fait-elle émerger de nouveaux territoires ?

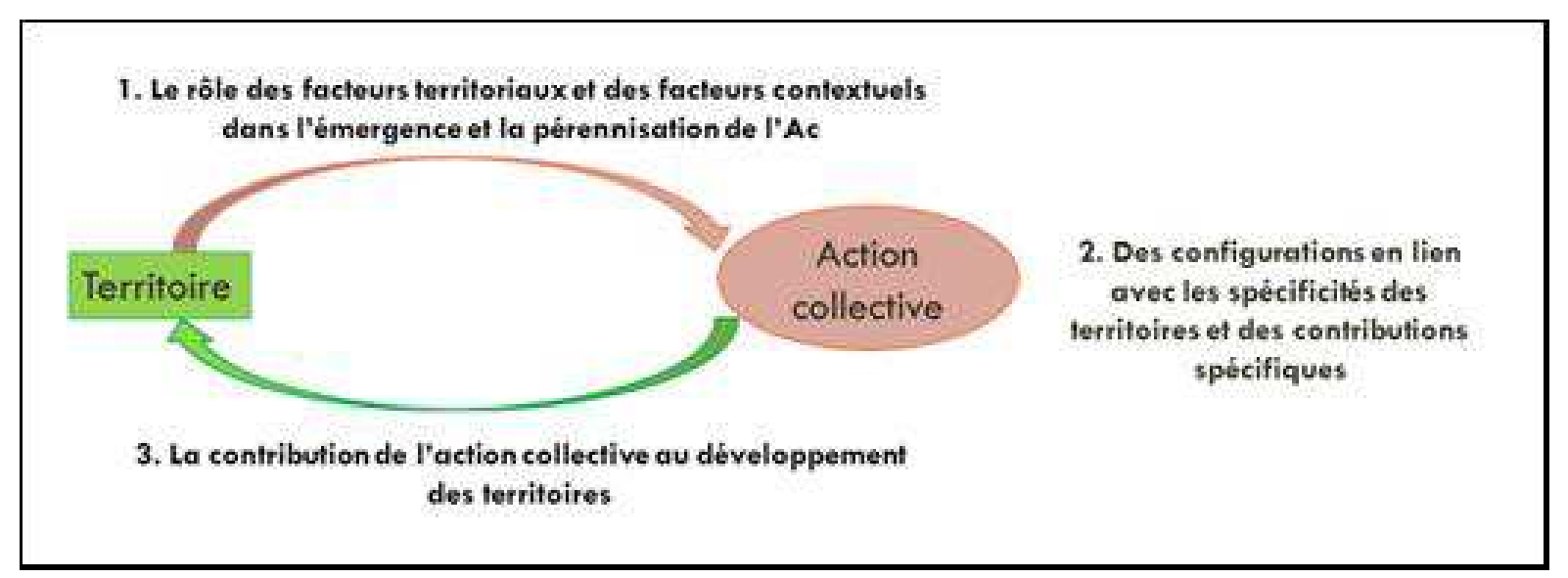

Figure 1: Les apports d'une entrée «territoire» de l'action collective: questions structurantes. 
Dans une première partie, nous présentons le positionnement des travaux analysés par rapport aux notions d'action collective et de territoire. Les parties suivantes exposent les apports d'une perspective territoriale de l'action collective sur les trois questions structurantes identifiées : les déterminants territoriaux de l'action collective (Partie 2), les formes d'action collective et la contribution spécifique de certains acteurs (Partie 3), et les conditions de construction de nouveaux territoires par l'action collective (Partie 4). La synthèse des travaux conduit à l'identification de perspectives en termes de recherche et d'accompagnement de l'action collective dans les territoires, perspectives qui sont développées en discussion.

\section{L'action collective dans une perspective territoriale : positionnement et définition}

De nombreuses disciplines ont produit différentes approches et concepts pour aborder l'action collective. En sociologie, les rôles, les arrangements, les justifications qui lient les différents acteurs dans les Ac sont particulièrement mis en exergue (Crozier et Friedberg, 1977 ; Friedberg, 1997 ; Callon, 1986 ; Boltanski et Thévenot, 1987 ; Latour, 1989). Les travaux en économie s'intéressent plus particulièrement aux intérêts des participants à l'Ac, qui fondent son existence et sa stabilité (Buchanan et Tullock 1962 ; Olson, 1965 ; Ostrom, 1990). En sciences politiques, les Ac sont mises en regard de systèmes ou logiques politiques vis-à-vis desquels elles prennent sens (March et Olsen, 1983; Tilly, 1985). Les sciences de gestion mettent en regard Ac et rationalité dans l'action (Hatchuel, 2001). Enfin, la géographie met l'accent sur la façon dont les pratiques sociales localisées construisent la conscience et l'identité sur laquelle repose l'action collective (Di Méo et Buléon, 2005 ; Lussault, 2007).

Dans les travaux s'intéressant à l'Ac en lien avec le territoire, les Ac peuvent s'inscrire dans des dynamiques qui n'excluent pas les conflits ou l'emprise d'intérêts extérieurs au territoire. Elles peuvent également ne pas être aussi formalisées que le voudrait la définition classique de Lorino (1989) : «L'action collective correspond à l'action commune ou concertée des 
membres d'un groupe en vue d'atteindre des objectifs communs ». Parfois il s'agira en effet d'un «mouvement d'essence plus ou moins spontanée, aux frontières incertaines et avec des structures fluides, émergentes et informelles, un mouvement qui représente le devenir, le surgissement du nouveau et l'historicité » (Friedberg, 1997). Ce complément de définition de l'Ac permet d'inclure les cas d'Ac diffus, informels, tout autant que les actions concertées très formelles. Nous retenons donc une définition de l'Ac située dans un gradient allant du mouvement diffus et spontané qui progressivement fédère plusieurs acteurs (par exemple, l'émergence de l'agriculture biologique sur un territoire) à l'action collective institutionnalisée (par exemple, les comités de bassin versant).

Dans une approche multidisciplinaire et multi-acteurs, le territoire est considéré comme le lieu d'articulation entre les politiques publiques et les initiatives locales, faisant de lui un niveau intermédiaire, entre le local et le global (Lardon et al., 2008). Il s'impose comme «lieu de coordination entre acteurs multiples, atomisés, en situation d'asymétrie, aux intérêts divergents et lieu de mise en cohérence d'objectifs divers, s'exprimant à des niveaux d'organisation enchâssés» (Gumuchian et al., 2003). Le territoire est conçu dans sa triple dimension matérielle, idéelle et organisationnelle (Laganier et al., 2002 ; Di Méo et Buléon, 2005 ; Moine, 2006). C'est un espace doté de propriétés naturelles résultant de l'aménagement de l'espace par les sociétés (dimension matérielle). Il est également caractérisé par son histoire et son patrimoine ainsi que par les représentations et les perceptions des groupes sociaux (dimension idéelle). Enfin, le territoire est défini comme une entité dotée d'une organisation de ses acteurs sociaux, politiques et institutionnels (dimension organisationnelle). Il est le lieu de dynamiques de développement territorial, au sens de «la capacité des acteurs à maîtriser les processus qui les concernent »(Deffontaines et al., 2001).

Plus précisément, la gamme des actions collectives auxquelles s'intéressent les travaux adoptant une perspective territoriale inclut : 
- Les Ac territoriales, c'est-à-dire des Ac dont la portée est transversale : toutes les composantes du territoire sont légitimes à prendre en considération. La question de ce que ces démarches apportent au territoire pour lui-même, dans ce qui le constitue comme référence signifiante, y est intrinsèquement posée.

- Les Ac qui se produisent à l'échelle du territoire mais n'ont pas la visée transversale des précédentes, ou ne sont pas étudiées pour cela. Les Ac sectorielles, qui se développent au sein de filières, ou encore les Ac concernant la gestion de ressources naturelles ou la fourniture de services environnementaux en constituent des exemples.

- Les Ac qui engagent des parties-prenantes et des enjeux locaux mais aussi des acteurs et enjeux relevant des échelles supra-territoriales. Elles ne renvoient pas à la construction du territoire en propre, comme les premières, mais peuvent néanmoins avoir des retombées tout à fait tangibles sur les territoires. On interroge alors leur ancrage territorial, ou leur territorialisation plus ou moins aboutie.

Les travaux réalisés sur des démarches d'Ac en lien avec le territoire se positionnent donc dans un spectre large de définitions de la notion. Dans les parties suivantes, nous mettons en évidence quelles sont les contributions de ces recherches à la compréhension des dynamiques territoriales.

\section{Le territoire, creuset d'actions collectives}

Les stratégies des acteurs impliqués, les procédures et modes de gouvernance mis en place, sont souvent au cœur de l'analyse du déploiement de l'action collective. L'analyse de l'action collective à l'échelle des territoires met en lumière comment des facteurs territoriaux conditionnent le développement de l'action collective (2.1.). Des travaux mettent également en évidence le rôle des facteurs liés aux contextes socio-économique et politique, au-delà des 
frontières du territoire, ainsi que les interactions entre caractéristiques du territoire et facteurs contextuels (2.2.).

\subsection{Les conditions territoriales de développement de l'action collective}

Les conditions territoriales du développement de l'action collective renvoient aux dimensions matérielles, organisationnelles et/ou idéelles des territoires.

Dans le champ de l'aménagement du territoire, la littérature a d'abord mis en avant les procédures de concertation mises en œuvre comme un facteur-clé de la réussite des projets (Mazeaud et al., 2012). Cependant, plusieurs travaux montrent désormais comment les caractéristiques mêmes des territoires peuvent peser sur le succès de la concertation collective (Bryson et Crosby, 1992 ; Ansell et Gash, 2007 ; Truffer et al., 2010 ; Berthomé et Thomas, 2017). Des variables propres à la dimension matérielle du territoire (d'ordre technique, comme la caractéristique des infrastructures) (Bulkeley et Askins, 2009) ou à sa dimension organisationnelle (dispositif de gestion en place, structure des incitations à coopérer) (Schusler et al., 2003 ; Daley, 2008), apparaissent ainsi déterminantes de l'issue des démarches collectives. Ces conditions territoriales (matérielles et organisationnelles) se combinent ainsi avec les conditions d'organisation de la concertation, de même qu'avec le ressenti des acteurs quant à la capacité et la fiabilité des équipements existants, l'historique de gestion (passif) et la gamme des options de gestion envisageables (Berthomé, 2014a).

Les travaux menés sur la gestion décentralisée des ressources naturelles ont initialement mis l'accent sur l'impact des systèmes de règles (modes de gouvernance) sur l'action collective associant les usagers des ressources (Schlager et Ostrom, 1992). Plus récemment, les recherches mobilisant le cadre des Systèmes Socio-Ecologiques (Ostrom, 2007 ; McGinnis et Ostrom, 2014) ont mis en évidence le rôle de la dimension matérielle du territoire, et plus précisément des caractéristiques de la ressource considérée dans la coopération entre acteurs 
(Agrawal, 2003 ; Thiel et al., 2012). Dans cette ligne, les travaux sur la coopération entre acteurs agricoles et collectivités locales pour la protection des captages d'eau potable montrent que la taille de l'aire d'alimentation de captage, le type de système hydrogéologique et le niveau de dégradation de la ressource en eau affectent l'émergence et la pérennisation de l'action collective (Barraqué et Viavattene, 2009 ; Bosc et Doussan, 2009 ; Amblard et Raynal, 2015).

Dans le champ de la production agricole, différents travaux mobilisant l'approche processuelle (Mendez et al., 2010) ont montré le poids de chacune des dimensions du territoire dans la mise en place et le développement des démarches collectives de différenciation et de valorisation des produits (Knickel et al., 2008 ; Schermer et al., 2011). L'analyse du cas de la valorisation de la viande bovine en Auvergne (Chabrat et al., 2014 ; Chabrat-Michel, 2015) montre qu'outre les effets conjugués des stratégies individuelles et collectives, les trois dimensions se combinent au fil de la trajectoire de l'action collective, chacune d'entre elles ayant une importance variable selon les temps du processus. Si les conditions matérielles en place (présence d'abattoirs, surface en herbe, nombre d'éleveurs) sont effectivement des éléments importants tout au long de l'action collective, les dimensions idéelles (valeurs culturelles, identité territoriale) et organisationnelles (structuration des acteurs autour d'une démarche AOP par exemple) prennent rapidement une importance majeure.

\subsection{Le rôle des facteurs liés aux contextes socio-économique et politique}

Plusieurs analyses critiques des travaux fondateurs de l'approche des «common-pool resources » ont souligné la faible attention portée au contexte externe par rapport au contexte local de l'action collective (Agrawal, 2003 ; Baron et al., 2011). Des travaux récents mettent en avant le rôle de l'Etat et des politiques publiques (Mansbridge, 2014) ainsi que l'incidence 
des évolutions socio-économiques globales (Baur et Binder, 2013) dans les dynamiques collectives locales.

Dans le cas de l'action collective associant acteurs agricoles et collectivités gestionnaires des captages d'eau potable, des facteurs liés au contexte politique et économique apparaissent jouer un rôle crucial (Bosc et Doussan, 2009). Plus particulièrement, l'existence de programmes d'appui à destination des maîtres d'ouvrage aux échelles départementale, régionale ou des bassins versants pallie, le cas échéant, aux contraintes pesant sur les collectivités gestionnaires en termes de ressources. Par ailleurs, les perspectives de valorisation économique des changements de pratiques agricoles induits par les démarches de protection de la ressource en eau jouent fortement sur la participation des agriculteurs aux démarches (Bosc et Doussan, 2009 ; Amblard et Raynal, 2015).

Le poids des contextes socio-économique et institutionnel dans l'émergence et le développement des actions collectives apparaît en filigrane des travaux portant sur les liens entre les dimensions agro-alimentaires et environnementales, sans être toujours directement analysé. Ainsi, le rôle des acteurs de «l'aval » (la grande distribution en particulier) est-il parfois abordé en tant que vecteur d'une demande sociale en matière de qualité des produits (Fontguyon et al., 2003 ; Burch et al., 2005 ; Baritaux et Houdart, 2015). Plus généralement, les différences de contextes sociaux et institutionnels semblent constituer un facteur de différenciation très important dans les spécificités des actions collectives allant dans le sens de la re-territorialisation des systèmes agro-alimentaires liés à l'élevage (Baritaux et al., 2016).

Ainsi, les travaux existants mettent en lumière la diversité des déterminants du développement des AC. Ces travaux soulignent le fait que conditions extra-territoriales et conditions territoriales sont toujours à l'œuvre pour expliquer le développement des actions collectives, 
parfois conjointement, parfois successivement, à différents temps du processus d'action collective. La multiplicité des facteurs intervenant dans le développement des Ac (internes au territoire et contextuels) met en évidence l'importance de la mobilisation de cadres conceptuels ou de modèles permettant d'appréhender le rôle joué par ces facteurs et leurs interactions. La prise en compte de la dimension temporelle apparaît également cruciale pour apprécier l'importance relative des facteurs territoriaux et extra-territoriaux dans les différents temps de l'action collective. La construction de cadres articulant l'ensemble des variables dans une perspective dynamique constitue de fait une piste de recherche à poursuivre.

\section{L'organisation des acteurs de l'action collective dans une perspective territoriale}

La diversité observée des formes prises par l'action collective dans les territoires pose la question des logiques sous-jacentes à ces configurations organisationnelles, en lien avec les enjeux de chaque territoire (2.1.). Rendre compte des contours des collectifs en jeu amène à interroger les rapports de pouvoir ou la construction de mondes communs entre les participants à l'Ac à l'échelle territoriale (2.2.). L'analyse de l'action collective dans les territoires révèle le rôle spécifique joué par certains acteurs en situation intermédiaire au sein des collectifs (2.3.).

\subsection{Des configurations liées aux enjeux territoriaux}

De nombreux travaux analysent la gouvernance de l'action collective et les facteurs qui contribuent à expliquer les formes organisationnelles observées (Agrawal, 2003 ; Kemper et al., 2007). Les travaux menés sur l'action collective à l'échelle des territoires permettent de structurer la diversité des formes observées, en lien avec les spécificités des territoires.

Différentes configurations organisationnelles de l'action collective pour l'entretien du paysage dans les espaces ruraux peuvent être ainsi distinguées en mobilisant le champ de 
l'économie des services (Gadrey, 2003). Ces configurations croisent le type d'acteur impliqué (collectivité territoriale, entreprise privée, association), le rôle joué par les acteurs dans la fourniture des services (commanditaire, prestataire, usager) et le type de bien-support des services (Aznar, 2002 ; Amblard et Déprés, 2011). Dans le cas de l'entretien de la voirie locale, trois configurations organisationnelles distinctes, correspondant à des logiques d'entretien différentes, ont été identifiées (Rambonilaza et al., 2013 ; Blasquiet-Revol et al., 2017). Dans la première configuration, les communes commanditent des services d'entretien en fonction des enjeux propres à leur territoire : agricole, résidentiel ou touristique. Dans la deuxième configuration, caractérisée par l'intervention des communautés de communes comme commanditaires, l'entretien de la voirie vise à favoriser le développement touristique du territoire intercommunal. Enfin, la troisième configuration, qui implique le conseil général, est dédiée aux loisirs récréatifs. L'analyse met en évidence le lien entre l'importance relative des configurations organisationnelles dans un territoire donné et les enjeux en termes d'usages et d'activités propres à ce territoire (Rambonilaza et al., 2013 ; Blasquiet-Revol et al., 2017).

Dans une perspective de développement territorial, les configurations socio-spatiales rendent compte des formes de l'action collective dans les territoires en lien avec les projets de territoire (Lardon et al., 2012). Dans le cadre de la labellisation des pôles d'excellence rurale², les formes d'organisation repérées diffèrent selon la répartition spatiale - concentrée ou diffuse - des acteurs et des actions dans le territoire de projet, et selon les liens - établis ou non - avec des acteurs extérieurs. La labellisation des pôles d'excellence rurale (PER) fournit un large panorama de la genèse des actions que mènent des acteurs locaux face aux enjeux de développement. Lardon et al. (2014) ont observé quelques cas de renforcement de dynamiques territoriales. Certains acteurs ont utilisé le dispositif PER pour impulser une

\footnotetext{
${ }^{2}$ Politique de la DATAR lancée en 2005 pour proposer un volet rural complémentaire aux pôles de compétitivité mis en place un an auparavant. Elle a fait l'objet d'une recherche évaluative en 2007-2009 d'où sont issus ces travaux.
} 
dynamique d'action collective qui se poursuit au-delà du financement direct des opérations (rôle intégrateur du dispositif). Le simple aboutissement d'une candidature a pu en soi constituer un résultat, dans le cas de démarches réellement partenariales révélant une capacité des acteurs territoriaux à se fédérer et à se coordonner pour porter un projet commun (rôle activateur). Pour certaines petites intercommunalités, la reconnaissance d'un PER a parfois constitué un premier acte de la légitimité de cet échelon administratif intercommunal à porter un projet de développement propre pour son territoire (rôle promoteur). La mise en place du dispositif a même servi de support à des formes d'innovation institutionnelle locale, en incitant des débats sur les recompositions profondes du territoire et les modalités d'organisation dont il se dote, comme lors de l'émergence d'un projet de PNR (rôle générateur). Dans l'ensemble, ces coordinations s'appuient sur des lieux et des moments de dialogue entre les différents acteurs impliqués dans la co-construction des démarches de développement. Elles s'appuient également sur des acteurs tels que ceux de la recherche et de la formation, ou encore les acteurs du conseil, qui n'étaient pas obligatoirement prévus au départ, mais qui émergent comme médiateurs (Cadoret, 2007) au cours du processus.

\subsection{Rapports de force et constitution d'une base d'adhésion à l'échelle du territoire}

L'étude de l'action collective ne tranche pas facilement la question de qui fait partie du collectif, notamment dans le cas des Ac qui engagent des parties prenantes extra-territoriales. Ainsi, dans le cas des décisions d'aménagement, les processus se centrent souvent sur le collectif des personnes décisives (Fourniau, 2007). Certaines parties prenantes plus influentes incarnent la ligne directrice de leur coalition (Berthomé, 2012). Dans le cas des projets de construction de territoire, où c'est la progression dans le domaine des idées, des représentations, qui importe (Debarbieux et Lardon, 2003), une large communauté débattante 
est visée. Le débat alimente les propositions d'actions, en amont, même si la décision finale n'est pas du ressort de tous.

Il y a une dynamique d'exploration, de partage, de circulation des questions dans le développement d'une Ac (Mormont, 2006), émaillée de mésententes ou d'incompréhensions. Ainsi, dans le cas des opérations de portage foncier visant la préservation de l'usage agricole ou l'accès à la terre, Léger-Bosch (2015) montre que les acteurs en présence ne partagent pas nécessairement les conventions auxquelles ils se réfèrent. De plus, ces conventions ne sont pas totalement stabilisées et leur évolution apparaît influencée par des jeux de pouvoir entre acteurs. Plus généralement, certaines tensions ou rapports de force entre les parties prenantes d'une Ac peuvent être difficilement surmontables (Ollivier-Trigalo et Rui, 2002). Berthomé (2014b) montre que dans le cas de concertations territoriales difficiles, l'équilibre comme le déséquilibre des rapports de force peuvent être des conditions qui favorisent l'acceptation de décisions collectives, les gains obtenus par les acteurs différant alors dans ces deux situations. Par ailleurs, dans le cas des Ac visant la valorisation du lien entre un produit et son territoire de production, Baritaux et Houdart (2015) mettent en évidence que, d'une manière générale, la mise en place d'une filière 'qualité' impliquant la grande distribution ne repose pas sur l'équilibre des pouvoirs ou des rapports de force entre les différents acteurs. Un déséquilibre effectif des rapports de force existe bien entre les différents acteurs, induit par le pouvoir de la grande distribution notamment. Mais la confiance et/ou l'ancienneté des relations, entre les producteurs et le transformateur ou le distributeur, constituent l'une des clefs d'aboutissement de la démarche.

En contrepartie, lorsqu'une vision fédératrice est proposée par un monde extérieur à celui des acteurs parties-prenantes de l'Ac, celle-ci peut peiner à se mettre en place, être mal reçue, voire détournée. Ainsi, dans le cas d'un réseau de saveurs mis en place par le PNR Livradois Forez, qui vise à renforcer le lien entre qualité des produits et territoire, dans une finalité de 
maillage et de développement territorial, le collectif de producteurs, transformateurs et distributeurs peine à se structurer en partie en raison de la difficulté à faire 'monde commun' (Fleury et al., 2014). Les acteurs de la production et ceux de la distribution sont freinés dans leurs interactions car ils connaissent mal les pratiques des uns et des autres, en termes d'organisation du travail en particulier.

\subsection{Des acteurs clés de l'action collective}

Les travaux d'Ostrom permettent d'identifier de grands principes qui sous-tendent les systèmes de gouvernance «robustes » pour la gestion collective d'une ressource commune, (Ostrom, 1990 ; Cox et al., 2010). Giest et Howlett (2014) insistent sur la modeste attention accordée dans ces travaux aux individus clés qui font circuler les informations, favorisent la réciprocité entre les membres des réseaux, font jouer les avantages de la collaboration. Au sein des Acs en lien avec le territoire, certains acteurs se distinguent par le service particulier qu'ils peuvent rendre au collectif. Trois formes de contributions spécifiques peuvent être identifiées : donner des garanties et contenir les dissensions, initier et favoriser le développement de réseaux d'acteurs, ou encore limiter les coûts de coordination.

Dans des démarches d'Ac décisives ayant une visée telle que la mise en place d'équipements publics, des acteurs décalés par rapport aux participants «partisans » contribuent à contenir les dissensions. Ils mènent un travail d'accommodement, qui peut consister à dégager des «garanties» des uns envers les autres. Ces acteurs peuvent avoir différentes charges publiques (sous-préfet, président de syndicat mixte de gestion par exemple, etc.). Ce qu'ils ont en commun est de chercher progressivement à dégager quelles actions, quels investissements, peuvent se réaliser (Berthomé, 2011). C'est ainsi que, pas à pas, les groupes de pression peuvent se ramener à des perspectives communes.

Dans certaines situations, des acteurs ayant une position relativement extérieure, ou plutôt « aux côtés » des parties prenantes engagées, se rendent utiles à la mise en lien des acteurs ou 
des enjeux. Par exemple, les PNR, considérés comme des acteurs territoriaux, jouent un rôle de «pivot» dans les territoires; ils ont un rôle intégrateur. Une des missions des PNR est notamment de concilier développement économique et protection de l'environnement sur la base d'une stratégie collective alliant acteurs économiques et acteurs de l'environnement. Dans le domaine du tourisme, la mobilisation du champ du management stratégique permet de caractériser une stratégie de mise en réseau d'entreprises pratiquée par certains PNR pour favoriser l'adhésion des opérateurs aux principes du développement durable (Marsat, 2011). Le PNR demeure extérieur au réseau d'entreprises, mais il peut servir successivement d'«architecte » (initiateur du réseau), puis de facilitateur (par exemple dans sa capacité d'apport de financement et de soutien logistique) (Marsat, 2007).

L'intervention d'un acteur facilitant l'Ac en lien avec le territoire ne repose pas uniquement sur les ressources dont il dispose et la place qu'il occupe structurellement sur le territoire. Cela a été mis en évidence dans le cas de l'organisation du service d'épandage agricole des boues d'épuration, service qui met en relation des collectivités locales avec des collectifs d'agriculteurs (Déprés, 2006 ; Déprés et Vollet, 2015). Ici, le facteur facilitant consiste à diminuer les coûts de coordination élevés que supportent les communes et qui limitent leur choix de l'option d'épandage agricole de boues d'épuration. Les recherches mettent en effet en évidence le rôle d'acteurs en situation d'intermédiaires dans la diminution des coûts d'organisation du service, sur la base d'une comparaison entre les départements du Puy-deDôme et de Haute-Vienne. L'existence d'un dispositif départemental de suivi des épandages en Haute-Vienne, reposant sur la chambre d'agriculture et un comité départemental de concertation, apparaît ainsi limiter les coûts d'accès à l'information des collectivités et des agriculteurs et les coûts de renégociation et de règlement des litiges, induisant une plus grande stabilité des conventions d'épandage (Amon et al., 2006 ; Déprés et al., 2008). 
Ainsi, la revue de littérature sur l'organisation des acteurs de l'action collective en lien avec le territoire met en avant le lien entre la diversité des configurations de l'Ac et les enjeux propres aux territoires dans lesquels elle s'inscrit. Sont également pointées, la difficulté à identifier les contours de certaines Ac, et les situations où des coalitions cherchent à peser sur les décisions. Enfin, les travaux révèlent les contributions spécifiques d'acteurs-clés au sein des collectifs (accommodement, intégration, facilitation). Ces contributions interviennent à différents moments et dans différentes situations, sans qu'elles ne soient nécessairement liées à un type d'acteur, de statut, ou de niveau d'organisation. Chaque type de contribution spécifique a probablement une certaine généricité. Il serait intéressant de voir dans quelle mesure chacun d'eux recoupe les types d'acteurs ou de rôles clés dans l'Ac tels qu'ils sont appréhendés dans la littérature classique sur le sujet (Goffman, 1974 ; Crozier et Friedberg, 1977) ou dans des travaux récents par exemple sur la médiation (Muller, 2005). Ce travail de croisement avec la littérature pourrait offrir un nouveau regard sur l'analyse des interactions entre acteurs.

\section{Quand l'action collective façonne les territoires}

La question de l'impact des Ac sur le territoire constitue le troisième volet majeur des travaux portant sur les relations entre Ac et territoire. Des recherches analysent les effets de l'Ac sur le développement économique des territoires (3.1) ; d'autres soulignent les effets indirects de l'Ac sur les transformations des territoires, par la création ou l'activation de nouvelles ressources (3.2).

\subsection{Action collective et développement économique des territoires}

La littérature s'intéressant aux effets de l'action collective sur le développement territorial considère principalement la dimension économique. 
Le modèle de développement territorial du «panier de biens » repose sur l'hypothèse que la valorisation conjointe de biens et services spécifiques peut permettre à certains territoires de dégager des avantages concurrentiels au travers de l'existence d'une rente territoriale (Pecqueur, 2001). Le panier de biens est le résultat d'un ensemble de biens et services complémentaires qui se renforcent les uns les autres sur les marchés locaux, d'une combinaison de biens (publics ou privés) participant de l'image du territoire et de ses effets de réputation et d'une coordination entre les producteurs du panier qui internalisent la rente territoriale (Angeon et Vollet, 2008). Les analyses menées dans différents territoires mettent en évidence que la pérennisation du modèle du panier de biens et de la rente territoriale dépend essentiellement des coordinations d'acteurs : coordination entre acteurs privés (accord sur périmètre d'origine et niveau d'exigence des cahiers des charges des produits du panier) et coordinations institutionnelles (lisibilité de l'accès aux biens et services du territoire), ainsi que de la cohérence entre action privée et action publique (Hirczak et al., 2008).

En ce qui concerne les politiques de développement territorialisées, les proximités géographique (distance supposée favoriser les relations entre acteurs) et organisée (capacités de coordination, capacité de décision collective, solidarité locale) sont considérés comme des atouts pour le développement économique des territoires (Rallet et Torre, 2004). Mathe et al. (2014) ont ainsi analysé les programmes Leader en Auvergne. Le programme Leader (Liaison entre actions de développement de l'économie rurale) repose sur des Groupes d'action locale (Gal) associant acteurs publics et privés, en charge de définir une stratégie locale de développement. Le développement de nouveaux partenariats porteurs d'innovations dans le cadre du programme Leader apparaît contraint par des stratégies de préservation des réseaux d'acteurs politico-administratifs existants, des contraintes administratives et financières ainsi qu'un risque de routinisation des actions affectant la plus-value innovante du programme (Bosc et Vollet, 2013). La mesure du degré de territorialisation des projets financés, en termes 
d'ancrage territorial, d'intersectorialité, de coordination entre acteurs et de transversalité, met en évidence la valeur ajoutée de la démarche Leader par rapport à des mesures mises en œuvre de manière plus « descendante», mais cette valeur ajoutée territoriale reste faible (Barbarot et al., 2013).

Les travaux mettent ainsi en évidence le rôle d'un certain nombre de facteurs relatifs à l'action collective dans le développement économique territorial : le type d'acteurs impliqués (coopération entre acteurs privés sectoriels ou intersectoriels, entre acteurs publics et privés), les stratégies individuelles des acteurs ainsi que les coûts associés à l'action collective.

\subsection{Action collective et création de nouvelles ressources}

Certaines recherches contribuent également à la mise en évidence des effets de l'action collective en termes de création de nouvelles ressources potentiellement activables pour le territoire. A ce titre, l'exemple de l'action collective menée autour de l'AOP fin gras du Mézenc apparaît particulièrement significative (Chabrat-Michel, 2015). L'auteure montre en effet qu'au-delà des ressources préexistantes à sa mise en place (ressource herbagère et floristique de qualité, pratiques et savoir-faire liés à la production et la commercialisation du Bœuf de Pâques), la démarche Fin Gras a aussi donné naissance à de nouvelles ressources territoriales telles que le produit lui-même, les fêtes ou encore les réseaux d'acteurs qui sont aujourd'hui la base de l'identité du Mézenc.

Dans le champ de la gestion des ressources naturelles, plusieurs travaux montrent que l'action collective peut contribuer au développement de ressources relationnelles (confiance, normes de réciprocité) pouvant être mobilisées dans le cadre de nouvelles démarches collectives (Lubell, 2007; Mandarano, 2009). Dans cette ligne, les travaux sur la coopération entre acteurs du secteur de l'eau et acteurs agricoles mettent en évidence que les contextes caractérisés par un historique d'animations collectives en lien avec la protection de la 
ressource en eau sont plus favorables à l'émergence de la coopération du fait de la plus grande interconnaissance entre acteurs (Barataud et al., 2013 ; Amblard et Reynal, 2015).

L'émergence de ressources relationnelles apparait également dans les dynamiques de développement rural. En particulier, les Acs qui s'inscrivent dans le cadre de la reterritorialisation des systèmes alimentaires sont vues par certains auteurs comme autant de reconfigurations des ressources et des réseaux de développement local (Watts et al., 2005 ; Chiffoleau et Prévost, 2012 ; Oosterveer et Sonnenfeld, 2012). Ainsi Loudiyi et al. (2014) ont-elles montré, dans le cas de l'analyse de la trajectoire d'une route de l'huile des monts pisans (Italie), le rôle qu'avait joué cette dynamique dans la construction de la capacité d'action des acteurs dans le contexte plus large d'un système agri-urbain. Ce projet a en effet permis aux acteurs partie-prenantes de renforcer leurs relations et, indirectement, leur pouvoir d'action dans des contextes de renouvellement des liens rural-urbain.

Ainsi, les travaux montrent comment l'Ac, participant au développement territorial dans une perspective économique ou de création de ressources, remodèlent le territoire. Cependant, l'évaluation des effets de l'Ac nécessiterait la prise en compte des interactions entre les multiples Ac co-existant dans le territoire. L'écheveau d'actions collectives, qui peuvent être concurrentes ou complémentaires, influe en effet sur leur capacité à transformer les territoires. Par ailleurs, qu'en est-il de la durabilité de l'impact des Acs sur le territoire ? Rares sont les travaux qui investissent théoriquement la question de la transformation dans la durée des territoires sous l'effet des actions collectives. Ce sont des pistes de recherche à poursuivre.

\section{Discussion : fronts de recherche}

La synthèse des travaux sur l'Ac dans une perspective territoriale a permis de mettre en évidence les apports de ce champ de recherche autour de trois principaux questionnements : 
les conditions de développement de l'Ac dans les territoires ; les acteurs et leur contribution à l'Ac dans les territoires; l'Ac comme moteur d'évolution des territoires. L'analyse permet également de dégager deux fronts de recherche transversaux (Fig.2). Le premier concerne la complexité des interactions entre Ac et territoire et l'intérêt de repenser les cadres conceptuels pour l'analyse de cette complexité (5.1.). Le second amène à s'intéresser aux démarches d'accompagnement de l'Ac pour ouvrir des perspectives en matière d'apports de connaissances pour l'action dans les territoires (5.2).

\subsection{Quels cadres conceptuels pour appréhender la complexité des interactions}

\section{Ac-territoire?}

L'intérêt de deux approches et de leur croisement peut être souligné : le cadre d'analyse des systèmes socio-écologiques et l'approche processuelle. Chacun de ces cadres d'analyses a été mis à l'épreuve de l'analyse de l'Ac dans les territoires, présentant des apports que nous pensons complémentaires.

Le cadre conceptuel des systèmes socio-écologiques (SSE) (Anderies et al., 2004 ; Ostrom, 2009) vise à élargir l'approche institutionnelle de l'action collective à la prise en compte des caractéristiques du système écologique considéré et des interactions avec les systèmes sociaux (Angeon et Caron, 2009). Il rassemble et structure un ensemble de facteurs susceptibles de jouer sur les interactions au sein de systèmes socio-écologiques et d'affecter leur durabilité. Quatre grandes catégories de variables sont distinguées : les caractéristiques de la ressource considérée, des acteurs impliqués dans la gestion de la ressource, les caractéristiques du système de gouvernance mis en place et le contexte social, économique et politique (Ostrom, 2007 ; 2009 ; McGinnis et Ostrom, 2014). Dans une démarche systémique, la mobilisation du cadre des systèmes socio-écologiques pour l'analyse de l'action collective dans un territoire 
permet d'appréhender à la fois le rôle des facteurs territoriaux, des modes de gouvernance et des facteurs contextuels. Cependant, si le cadre se donne pour objectif l'analyse des dynamiques des systèmes socio-écologiques, les modalités de prise en compte des évolutions des différents sous-systèmes et des effets rétroactifs restent à expliciter (Anderies et Janssen, 2013 ; Hinkel et al., 2015).

Moins robuste sur la prise en compte du contexte institutionnel et de l'état initial de la ressource à gérer, l'approche processuelle (AP) apparaît complémentaire du cadre des SSE en particulier en introduisant la possibilité d'identifier les ressources territoriales activées au fil du temps. Dans une perspective dynamique, le cadre de l'AP vise à avoir une vision globale, holistique, des faits sociaux en ayant une approche constructiviste du temps (Oiry et al., 2010). Les relations et modes d'organisation entre acteurs sont ainsi le résultat de trajectoires qui correspondent à un « enchaînement de faits dans le temps aboutissant à un résultat, ou un phénomène conçu comme actif et organisé dans le temps » (Mendez et al., 2010). Le cadre d'analyse processuelle vise ainsi à comprendre la combinaison des dynamiques qui fondent les processus (Pettigrew, 1997). Ces derniers se caractérisent par quatre composantes. Les ingrédients sont les éléments du contexte global dans lequel se déroule le processus qui apparaissent pertinents pour comprendre ce dernier : les identifier peut revenir à distinguer les conditions territoriales mobilisées par l'action ainsi que les conditions supra-territoriales influençant l'action. Les séquences sont les segments temporels qui s'enchaînent et forment le processus. Les moteurs sont définis comme des mécanismes génératifs du mouvement des ingrédients et de leur assemblage au cours du temps : leur analyse aboutit à la compréhension des modes de coopérations, aux relations entre acteurs, introduisant notamment la possibilité de considérer les conflits comme moteur de l'action. Les bifurcations correspondent à un changement dans l'assemblage des ingrédients, modifiant l'orientation même du processus. Cette approche invite le chercheur à prendre en compte les interactions entre les différents 
épisodes et à éviter de considérer le processus comme linéaire et prédéterminé. Elle introduit l'analyse des trajectoires, pour aller plus loin que les seules configurations socio-spatiales. En contrepartie, elle invite le chercheur à s'intéresser à des Ac ayant une certaine profondeur historique et exclut, de fait, l'analyse d'Ac émergentes.

Outre la prise en compte de la multiplicité des facteurs territoriaux et supra-territoriaux jouant sur le développement de l'Ac, le croisement du cadre SSE et de l'approche processuelle nous semble également pouvoir apporter des éléments de réponse à la question de l'impact de l'Ac sur les territoires au travers de la caractérisation dans le temps des effets de l'Ac sur les caractéristiques matérielles (ressources naturelles et construites), organisationnelles (interactions entre acteurs, gouvernance) et idéelles (représentations des acteurs) du territoire. En effet, la mobilisation de l'approche processuelle, explicitement dynamique, peut permettre une opérationnalisation des effets rétroactifs de l'action collective, envisagés dans le cadre des SSE, sans avoir été explorés dans les applications du cadre jusqu'à présent.

Deux modalités nous semblent possibles pour mettre à l'épreuve le croisement de ces deux cadres d'analyse : l'hybridation ou la juxtaposition, tout en étant attentif à la possible perte du pouvoir analytique de chacun de ces cadres s'ils sont croisés. L'hybridation consisterait à croiser pas à pas les deux approches, les catégories de variables du cadre des SSE s'exprimant en termes d'ingrédients et de moteurs de l'AP et la dynamique de l'AP renvoyant, d'une étape à la suivante, à un SSE transformé. Cela semble cependant une démarche assez lourde à mener. La juxtaposition consisterait à mobiliser côte à côte les deux démarches, ce qui simplifierait l'analyse, en termes de moyens et d'engagement dans le temps, cela, au risque de perdre la lecture très intégrée du développement des Ac dans le territoire que permet l'hybridation. 


\subsection{Quels outils d'accompagnement de l'Ac dans les territoires pour quelle}

portée heuristique ?

Une posture de recherche-action peut offrir une autre lecture des trois questionnements structurant les apports des travaux sur l'action collective en lien avec le territoire : Comment permettre l'émergence et le développement de l'Ac ? Comment assurer l'adhésion des partiesprenantes et légitimer le rôle des acteurs contribuant de façon significative à l'Ac ? Comment accompagner, prévoir, penser les effets de l'Ac sur les territoires?

Des expériences de démarches participatives permettent d'identifier les éléments de réponse déjà apportés à ces questions et les points restant à instruire.

Par exemple, le « jeu de territoire » est un jeu d'expression qui favorise la construction d'une vision partagée du territoire et répond à trois principes (Lardon, 2013). Il constitue une recherche-intervention, au sens d'Hatchuel (2001), apportant sa capacité de prise de recul et de formalisation. La production des connaissances pour l'action se fait dans un processus itératif où les savoirs des acteurs et ceux des chercheurs sont mis en commun (Beguin, 2007) et appropriés. Il y a conception collaborative (Brassac, 2004) où chacun se retrouve et tire parti de la production collective. Ainsi, le jeu permet l'établissement d'un dialogue entre acteurs du territoire (élus, habitants, professionnels...) et chercheurs, autour de différents objets intermédiaires (Vinck, 2009) tels que les cartes produites au cours du processus de production de connaissances et de valorisation des expériences.

De telles démarches participatives, permettent d'accompagner la conception d'actions collectives, qui renforcent la capacité des acteurs à mettre en œuvre leur projet de territoire (constitution d'une intercommunalité ou d'un PNR, planification territoriale (SCoT, PLU), gestion des espaces agricoles périurbains) (Lardon, 2015). Elles permettent tout à la fois de 
comprendre les dynamiques d'évolution des territoires ruraux et périurbains et d'accompagner les acteurs du changement dans la conception, la réalisation et l'évaluation de leurs projets de territoire (Lardon et al., 2007). Ainsi, ces démarches informent à la fois la question de l'accompagnement dans le développement de l'Ac et celle des effets de l'Ac sur les territoires. La portée heuristique de ces démarches est assurée par une posture réflexive (Trognon, 2013), tant sur les interactions chercheurs-acteurs dans la conduite de projets territoriaux que sur les modalités de montée en compétence pour la mise en œuvre d'actions collectives.

De plus, situées en amont des décisions, ces démarches facilitent l'engagement des acteurs tout en renseignant la question des blocages et des conflits entre acteurs qui peuvent freiner l'Ac dans les territoires. Cela peut promouvoir des modalités de rencontres collaboratives (les chercheurs ignorants, 2015) à même de pallier les tensions souvent attisées par la proximité de décisions, engageantes, perçues comme irréversibles (Rui, 1999). C'est ainsi que peut se créer une mise en commun qui fasse sens, un assemblage nouveau entre des éléments épars, un «monde commun » (Stengers, 2006).

Une question reste à instruire dans le cadre de ces démarches : comment légitimer le rôle des acteurs contribuant de façon significative à l'Ac ? Si nous avons identifié les contributions spécifiques que peuvent apporter certains acteurs en situation intermédiaire, nous avons considéré des dispositifs distincts, sans analyser en retour comment les politiques publiques pourraient contribuer à soutenir (ou ne pas entraver) ces contributions. Comment professionnaliser ces acteurs en tant que médiateurs (Cayre et al., 2014) pour qu'ils investissent le champ du développement territorial?

Finalement, ces démarches participatives permettent tout à la fois de comprendre et d'accompagner des changements ; elles éclairent les modalités d'une gouvernance territoriale. Parce que les acteurs porteurs sont impliqués et s'approprient les démarches, parce que les 
actions collectives mettent en relation les incitations politiques et les initiatives locales, les démarches participatives de projet de territoire deviennent légitimes (Barnaud, 2013). Elles sont les supports d'interdisciplinarité et de transdisciplinarité nécessaires à la prise en compte de systèmes complexes (Borderon et al., 2015).

\section{Conclusion}

Dans cet article, nous mettons en avant les apports d'une entrée « territoire » de l'action collective sur la base d'une revue de la littérature et de cas d'étude récents dans les domaines de l'aménagement et du développement des territoires ruraux et périurbains, de la gestion des ressources naturelles, de l'agriculture et de l'alimentation. Le croisement de ces domaines, des regards de la géographie et de l'économie, apporte une richesse de points de vue et d'expériences. Cela permet également de mettre en évidence l'opportunité d'une intégration des connaissances pour la prise en compte de la complexité des interactions entre action collective et territoire.

Les apports des recherches sur l'Ac dans une perspective territoriale renseignent trois grandes questions structurantes. Tout d'abord, au-delà des déterminants classiques de l'action collective (jeux d'acteurs, modes de gouvernance), les travaux révèlent l'incidence des conditions locales, en interaction avec des facteurs contextuels. Ensuite, les recherches mettent en évidence le lien entre configurations d'action collective dans les territoires et enjeux territoriaux, la construction de mondes communs à l'échelle des territoires et les contributions spécifiques de certains acteurs. Enfin, les travaux montrent comment l'action collective produit en retour de nouvelles dynamiques, de nouvelles ressources, de nouvelles perspectives pour le territoire. L'analyse des recherches conduit à l'identification de deux fronts de recherche majeurs. L'un porte sur les croisements entre les cadres conceptuels socioécologiques et processuels, pour traiter la complexité et la temporalité de l'Ac en lien avec le 
territoire. L'autre concerne l'accompagnement de l'action collective dans les territoires, s'appuyant sur des démarches participatives engageant les acteurs parties-prenantes.

La synthèse proposée pourrait être enrichie par deux perspectives complémentaires. D'une part, bien que la dimension idéelle du territoire soit prise en compte dans certains travaux, nous avons peu exploré les dimensions culturelles et sensibles qui président à l'engagement dans des actions collectives. L'attachement au territoire, ou « sense of place », oriente les Acs, car il est alimenté par un vécu, un rapport direct au territoire, qui peut constituer une terre d'appartenance, voire de subsistance (Chapin et Knapp, 2015). Des approches anthropologiques ou historiques auraient ici leur place. D'autre part, un point de vue ancré en science politique pourrait éclairer de manière complémentaire des problématiques telles que les contributions spécifiques de certains acteurs à l'Ac, l'adhésion des parties prenantes ou les rapports de force qui s'expriment dans l'Ac. Cette perspective permettrait de mieux appréhender l'inscription de ces phénomènes dans le cadre de politiques publiques organisées, ainsi que le sens de l'action pour les différents acteurs, notamment les acteurs politiques. La question des instruments/outils politiques et des modes d'accompagnement publics de l'action collective dans les territoires pourrait être également traitée spécifiquement. Finalement, les fronts de recherche proposés ouvrent des perspectives en matière d'apports de connaissances sur les interactions entre Ac et territoire, en posant la question du croisement des cadres théoriques et de l'accompagnement de l'action dans les territoires. Nous les mettons à l'agenda des futures recherches.

\section{Références bibliographiques}

Adger W. N., 2003. Social capital, collective action and adaptation to climate change. Economic Geography 79/4, 387-404. 
Agrawal A., 2003. Sustainable Governance of Common-Pool Resources: Context, Methods, and Politics. Annual Review of Anthropology 32, 243-262.

Amblard L., Déprés C., 2011. La filière paysage en milieu rural. Une analyse de l'organisation des services d'entretien à dimension paysagère, in Analyses économiques du paysage, coordonné par Oueslati W., Paris, Editions QUAE, 117-130.

Amblard L., Reynal V., 2015. La coopération entre producteurs d'eau potable et acteurs agricoles en France. Les conditions d'émergence et de pérennisation de l'action collective. Rapport final convention de recherche Irstea-Onema 2013-2015, 137 p.

Amon G., Aznar O., Vollet, D., 2006. Why are some French farmers sludge-takers? Some agronomic and socioeconomic explanations. International Journal of Agricultural Resources, Governance and Ecology 5 (2/3), 289-308.

Anderies J.M., Janssen M.A., 2013. Robustness of Social-Ecological Systems: Implications for Public Policy. Policy Studies Journal, 41/3, 513-536.

Anderies J.M., Janssen M.A., Ostrom E., 2004. A framework to analyse the robustness of socialecological systems from an institutional perspective. Ecology and Society 9, 1-18.

Angeon V., Caron A., 2009. Quel rôle joue la proximité dans l'émergence et la pérennité de modes de gestion durable des ressources naturelles ? Natures Sciences Sociétés 17, 361-372.

Angeon V., Vollet D., 2008. Spécificité des produits et développement territorial. L'exemple paradoxal du panier de biens en émergence de l'Aubrac. Revue d'Economie Régionale et Urbaine 4, 591-615.

Ansell C., Gash A., 2007. Collaborative Governance in Theory and Practice. Journal of Public Administration Research and Theory 18, 543-571. 
Aznar O., 2002. Services environnementaux et espaces ruraux. Une approche par l'économie des services. Thèse de Doctorat, Université de Bourgogne, Département d'Economie, 273 p.

Barataud F., Aubry C., Wezel A., Mundler P., 2013. Management of drinking water catchment areas in cooperation with agriculture and the specific role of organic farming. Experiences from Germany and France. Land Use Policy 36, 585-594.

Barbarot A.S., Renard T., Vollet D., Lépicier D., 2013. Propositions méthodologiques pour mesurer la territorialisation des projets de développement rural. Economie Rurale 336, 81-88.

Baritaux V., Houdart M., 2015. Relations fournisseurs-grande distribution dans les filières agroalimentaires. Une analyse de la trajectoire d'une démarche « filière qualité ». Économie Rurale 346, 15-30.

Baritaux V., Houdart M., Boutonnet J.P., Chazoule C., Corniaux C., Fleury P., Lacombe N., Napoléone M., Tourrand J.F., 2016. Ecological Embeddedness in Animal Food Systems (Re)Localisation: A Comparative Analysis of Initiatives in France, Morocco and Senegal. Journal of Rural Studies 43, 13-26.

Barnaud C., 2013. La participation, une légitimité en question. Natures Sciences Sociétés 21, 24-34.

Baron C., Petit O., Romagny B., 2011. Le courant des «Common-Pool Resources » : un bilan critique, in: Dahou T., Elloumi M., Molle F., Gassab M., Romagny B. (dir.). Pouvoirs, Sociétés et Nature au Sud de la Méditerranée, Paris/Tunis. Editions INRAT/IRD/Karthala, 2951.

Barraqué B., Viavattene C., 2009. Eau des Villes et Eau des Champs. Vers des accords coopératifs entre services publics et agriculteurs ? Economie Rurale 310, 5-21. 
Baur I., Binder CR, 2013. Adapting to socio-economic developments by changing rules in the governance of common property pastures in the Swiss Alps. Ecology and Society 18/4, http://hdl.handle.net/10535/9217

Beguin P., 2007. Innovation et cadre socio-cognitif des interactions concepteurs-opérateurs : une approche développementale. Le Travail Humain 7/4, 369-390.

Berthomé G-E-K., 2011. Un peu de diplomatie et beaucoup de résignation: La décision concertée dans tous ses états, Journée d'étude sur les effets de la participation du public aux processus décisionnels, octobre 2011, Paris.

Berthomé G-E-K., 2012. Les préférences des participants aux concertations sur les déchets selon une méthode non déclarative. Colloque International sur la prévention et la gestion durable des déchets, novembre 2012, Le Mans.

Berthomé G-E-K., 2014a. Simuler les résultats des concertations environnementales. Négociations 2/22, 53-68.

Berthomé G-E-K., 2014b. Narrowing down the negotiation power issue in stakeholders dialogue by measurement. Policy \& Politics conference 2014, 16 -17 September 2014, University of Bristol, UK.

Berthomé, G.-E.-K., Thomas, A., 2017. A Context-based Procedure for Assessing Participatory Schemes in Environmental Planning. Ecological Economics 132, 113-123.

Blasquiet-Revol, H., Amblard, L., Aznar, O., Déprés, C., 2017, Organisation de l'entretien de la voirie locale dans les territoires ruraux. Le cas de trois communes du Puy-de-Dôme, Economie Rurale 360, à paraître. Boltanski L., Thévenot L., 1987. Les Economies de la grandeur. Cahiers du Centre d'étude de l'emploi 31, PUF, Paris. 
Borderon M., Buchs A., Leblan V., Vecchione E., 2015. Réflexivité et registres d'interdisciplinarité. Une boussole pour la recherche entre natures et sociétés. Natures Sciences Sociétés 23/4, 399-407.

Bosc C., Vollet D., 2013. Évaluation du dernier programme Leader (2007-2013) en Auvergne et Bourgogne : entre logiques de routinisation et risque de capture agricole. Politiques et Management Public 30/4, 473-492.

Bosc C., Doussan I., 2009. La gestion contractuelle de l'eau avec les agriculteurs est-elle durable ? Approche politique et juridique. Economie Rurale 309, 65-80.

Brassac C., 2004. Action située et distribuée et analyse du discours : quelques interrogations. Cahiers de linguistique française 26, 251-268.

Bryson J.M., Crosby B.C., 1992. Leadership for the common good: Tackling public problems in a shared-power world. Jossey-Bass, San Francisco.

Buchanan J.M., Tullock G., 1962. The calculus of consent (Vol. 3). Ann Arbor, University of Michigan Press.

Bulkeley H., Askins K., 2009. Waste interfaces: biodegradable waste, municipal policy and everyday practice. Geographical Journal 175/4, 251-260.

Burch D., Lawrence G., 2005. Supermarket Own Brands, Supply Chains and the Transformation of the Agri-Food System. International Journal of Sociology of Agriculture and Food 13/1, 1-18.

Cadoret A., (2007) « la médiation : outil pour une gestion durable des conflits d'usage ?» Colloque Développement Durable et Gouvernance desTerritoires, ERSA et ASRDLF, Paris, 29 aout-2 septembre 2007. 
Callon M., 1986. Some elements of sociology of translation: domestication of the scallops and the fishermen of St Brieuc Bay, in Law (ed.), "Power, action and belief: a new sociology of knowledge", Routledge, London.

Cayre P., Chambon P., Trognon L., 2014. Ingénierie territoriale, formation et processus de socialisation. In Angeon V., Lardon S., LeBlanc P., (dir.), Compétences, formation et apprentissage collectif territorial, Tome 1, L'Harmattan, 85-130.

Chabrat-Michel S., 2015. Impacts des conditions territoriales sur la mise en place et le développement des démarches collectives de valorisation de la viande bovine en zones de moyenne montagne auvergnate. Thèse de doctorat de géographie de l'Université BlaisePascal, 345p.

Chabrat S., Baritaux V., Houdart M., 2014. De la viande, du foin et un pastoralisme sédentaire, Le cas de l'AOP Fin Gras du Mézenc. Revue de géographie alpine 102/2, http://rga.revues.org/2422.

Chapin F.S., Knapp C.N., 2015. Sense of place: A process for identifying and negotiating potentially contested visions of sustainability. Environmental Science and Policy 53, 38-46.

Chiffoleau Y., Prevost B., 2012. Les circuits courts, des innovations sociales pour une alimentation durable dans les territoires. Norois 224, 7-20.

Cox, M., Arnold, G., Villamayor Tomas, S., 2010, A review of Design principles for Community-based Natural Resource Management, Ecology and Society 15 (4): 38.

Crozier M., Friedberg E., 1977. L'acteur et le système : les contraintes de l'action collective. Seuil, Paris. 
Daley D.M., 2008. Interdisciplinary Problems and Agency Boundaries: Exploring Effective Cross-Agency Collaboration. Journal of Public Administration Research and Theory 19, 477 493.

Debarbieux B., Lardon S. (dir.) 2003. Les figures du projet de territoire. Editions de l'Aube, La Tour d'Aigues.

Deffontaines J.P., Marcelpoil E., Moquay P., 2001. Le développement territorial : une diversité d'interprétations, in Lardon S., Maurel P., Piveteau V. (dir.), Représentations spatiales et développement territorial. Hermès, Paris, p. 39-56.

Déprés C., Aznar O., Vollet D., Jeanneaux P., 2008. Identification et notion de coûts d'organisation dans les services collectifs locaux. Étude de cas : le service d'épandage agricole des boues d'épuration. Ingénieries - EAT 54, 33-41.

Déprés, C. et Vollet, D., 2015, La gestion du changement institutionnel en matière d'épandage agricole des boues d'épuration en France, Territoire en mouvement, 25-26, URL : http://tem.revues.org/2730 ; DOI : 10.4000/tem.2730.

Déprés, C., 2006, Une approche néo-institutionnelle de la fourniture de services environnementaux : le cas du service d'épandage agricole des boues d'épuration et ses formes d'organisation territoriale, Thèse de doctorat en sciences économiques, Université de Bourgogne, 305 pages.

Di Méo G., Buléon P., 2005. L'espace social : lecture géographique des sociétés. Armand Colin, Paris.

Dionnet M., Guérin-Schneider L., 2014. La coordination inter-organisationnelle, levier de la gouvernance territoriale : quelles leçons tirer de la gestion de l'eau interbassin? Géographie, économie, société 16/4, 399-420. 
Dubuisson-Quellier S., 2001. Une organisation territoriale pour la mise en place d'une filière porc de qualité en Bretagne. Etud. Rech. Syst. Agraires Dév. 32, 165-176.

Fleury P., Houdart M., Lasseur J., Linck T., 2014. Diversité des actions collectives en élevage et des formes d'intensification écologiques qu'elles expriment. Livrable 2.3, projet Mouve, https://www1.clermont.inra.fr/mouve/index.php?p=productions\&loc=Les\%20Productions.

De Fontguyon G., Giraud-Héraud E., Rouached L., Soler L.G., 2003. Qualité des produits alimentaires et marques de filières. Sociologie du Travail 45/1, 77-94.

Fourniau J.M., 2007. "Citoyen en tant que riverain" : une subjectivation politique dans le processus de mise en discussion publique des projets d'aménagement, in Revel M., Blatrix C. Blondiaux L., Fourniau J. M., Hériard-Dubreuil B., Lefebvre R. (eds), Le débat public : une expérience française de démocratie participative. La Découverte - Recherches, Paris, 67-77.

Friedberg E., 1997. Le pouvoir et la règle. Dynamiques de l'action organisée. Seuil, Paris.

Gadrey J., 2003. Socio-économie des services. Repères, la Découverte, Paris.

German L., Hailemichael T., 2008. A framework for evaluating effectiveness and inclusiveness of collective action in watershed management. J. Int. Dev. 20, 99-116.

Giest S., Howlett M., 2014. Understanding the pre-conditions of commons governance: The role of network management. Environmental Science \& Policy 36, 37-47.

Goffman E., 1974. Frame analysis: An essay on the organization of experience. University Press, Harvard.

Goldman R.L., Thompson B.H., Daily G.C., 2007. Institutional incentives for managing the landscape: Inducing cooperation for the production of ecosystem services. Ecological Economics 64, 333-343. 
Gumuchian H., Grasset E., Lajarge R., Roux E., 2003. Les acteurs, ces oubliés du territoire. Economica, Paris.

Hatchuel A., 2001. Quel horizon pour les sciences de gestion? Vers une théorie de l'action collective, in David, Hatchuel, Laufer (eds.), Les nouvelles fondations des sciences de gestion. Vuibert, Paris, 21-45.

Hinkel J., Cox M., Schlüter M., Binden C.R., Falk T., 2015. A diagnostic procedure for applying the social-ecological systems framework in diverse cases. Ecology and Society 20/1, 32.

Hirczak M., Moalla M., Mollard A., Pecqueur B., Rambonilaza M., Vollet D., 2008. Le modèle du panier de biens. Grille d'analyse et observations de terrain. Economie Rurale 308, $55-72$.

Hugues F., Lirczac M., Senil N., 2013. De la ressource à la trajectoire : quelles stratégies de développement territorial ? Géographie, économie, société 15/3, 267-284.

Kemper, K.E., Blomquist, W., Dawson, J., Dinar, A., 2007. Integrated river basin management through decentralization. Springer, Berlin.

Knickel K, Zerger C., Jahn G., Renting H., 2008. Limiting and Enabling Factors of Collective Farmer's Marketing Initiatives: Results of a Comparative Analysis of the Situation and Trends in 10 European Countries. Journal of Hunger \& Environmental Nutrition 3/2, 247-269.

Laganier R., Villalba B., Zuindeau B., 2002. Le développement durable face au territoire: éléments pour une recherche pluridisciplinaire. Développement durable et territoires 1 , http://developpementdurable.revues.org/774.

Lardon S., 2013. Le « jeu de territoire », un outil de coordination des acteurs locaux. Revue FaçSADe 38, 4p. 
Lardon S., 2015. L'agriculture comme potentiel de développement des territoires péri-urbains. Analyse par les configurations socio-spatiales. Articulo - Journal of Urban Research 6, http://articulo.revues.org/2673.

Lardon S., Moquay P., Poss Y., 2007. Développement territorial et diagnostic prospectif: réflexions autour du viaduc de Millau, Editions de l'Aube, Paris, France, 377p.

Lardon S., Loudiyi S., Cournut E., Fournier J., 2012. Les configurations socio-spatiales : outil et dispositif de gouvernance des territoires. Revue d'Auvergne 602-603, 295-310.

Lardon S., Milian J., Loudiyi S., Leblanc P., Barthe L., Taulelle F., 2014. Du potentiel à l'action : la gouvernance territoriale des pôles d'excellence rurale. Norois 233/4, 69-81.

Lardon S., Tonneau J.P., Raymond R., Chia E., Caron P., 2008. Dispositifs de gouvernance territoriale durable en agriculture. Norois 209/4, 17-36.

Latour B., 1989. La science en action. La Découverte, Paris.

Léger-Bosch C., 2015. Les opérations de portage foncier pour préserver l'usage agricole : une analyse par les coordinations, les transactions et les institutions. Thèse de doctorat en économie, Université Grenoble Alpes.

Les Chercheurs ignorants (coord.), 2015. Les recherches-actions collaboratives, Une révolution de la connaissance, Presses de l'EHESP, 279 pages.

Lévy J., Lussault M., 2003. Dictionnaire de la géographie et de l'espace des sociétés. Belin, Paris.

Lindberg G., Fahlbeck E., 2011. New forms of local collective governance linked to the agricultural landscape: identifying the scope and possibilities for hybrid institutions. Int. J. Agricultural Ressources, Governance and Ecology 9/1-2, 31-47.

Lorino P., 1989. L'économiste et le manageur. La Découverte, Paris. 
Loudiyi S., Houdart M., Giacché G., 2014. Action collective et dynamiques agri-urbaines : le cas de la route de l'huile du monte pisano (Pise, Italie), in Colloque international de géographie rurale "Les campagnes : espaces d'innovation dans un monde urbain", 2 au 6 juin Nantes.

Lubell M., 2007. Familiarity breeds trust: Collective action in a policy domain. Journal of Politics 29/1, 237-250.

Lubell M., Schneider M., Scholz J.T., Mete M., 2002. Watershed partnerships and the Emergence of Collective Action Institutions. American Journal of Political Science 46/1, 148163.

Lussault M., 2007. L'homme spatial : La construction sociale de l'espace humain. Le seuil, collection « La couleur des idées », Paris.

Mandarano L., 2009. Social Network Analysis of Social Capital in Collaborative Planning. Society \& Natural Resources 22/3, 245-260.

Mansbridge J., 2014. The role of the state in governing the commons. Environmental Science and Policy 36, 8-10.

March J.G., Olsen J.P., 1983. The new institutionalism: organizational factors in political life. American political science review 78/3, 734-749.

Markelova H., Meizen-Dick R., Hellin J., Dohrn S., 2009. Collective action for smallholder market access. Food Policy 34, 1-7.

Marsat J.B., 2007. Le réseau, un objet de gestion stratégique pour des dynamiques territoriales de développement durable : applications en tourisme. XLIII Colloque de l'ASRDLF - Les dynamiques territoriales, débats et enjeux entre les différentes approches disciplinaires. Chambéry, 11-13 Juillet 2007. 
Marsat J.B., 2011. L'action des PNR pour un tourisme durable : stratégies et outils, une analyse dialogique. 2èmes journées scientifiques du tourisme durable, Poitiers, France, 23/06/2011.

Mathe J., Vollet D., Lépicier D., Berriet-Solliec M., Le Roy A., 2014. Évaluation régionale LEADER en Auvergne : un bilan mitigé et contrasté en termes de valeur ajoutée. Sciences Eaux et Territoires 13, 38-43.

Mazeaud A., Sa Vilas Boas M.H., Berthomé G-E-K., 2012. Penser les effets de la participation sur l'action publique à partir de ses impensés. Participations 1/1, 5-29.

McGinnis M.D., Ostrom E., 2014. Social-ecological system framework: initial changes and continuing challenges. Ecology and Society 19/2, 30.

Meinzen-Dick R., DiGregorio M., McCarthy N., 2004. Methods for studying collective action in rural development. Agricultural Systems 82, 197-214.

Mendez A., Bidart C., Brochier D., Correia M., Garnier J., Gilson A., Longo M.-E., Mercier D., Oiry E., Pascal A., Perocheau G., Tchobanian R., 2010. Processus : Concepts et méthode pour l'analyse temporelle en sciences sociales, Academia-Bruylant, Paris.

Moine A., 2006. Le territoire comme un système complexe: un concept opératoire pour l'aménagement et la géographie. L'Espace géographique 35, 115-132.

Muller, P., Esquisse d'une théorie du changement dans l'action publique. Structures, acteurs et cadres cognitifs, Revue française de science politique, 55 :155-187.

Mormont M., 2006. Conflit et territorialisation. Géographie, Economie, Société 8, 299-318.

Oiry E., Bidart C., Brochier D., Garnier J., Gilson A., Longo M.E., Mendez A., Mercier D., Pascal A., Perocheau G., Tchobanian R., 2010. Propositions pour un cadre théorique unifié et 
une méthodologie d'analyse des trajectoires des projets dans les organisations. Management \& Avenir 6/36, 84-107.

Ollivier-Trigalo M., Rui S., 2002. L'expertise comme outil d'évaluation et de communication. Metropolis 108/109, 84-87.

Olson M., 1965. The logic of collective action. Public goods and the theory of groups. Harvard University Press, Cambridge.

Oosterveer P., Sonnenfeld, D., 2012. Food, Globalization and Sustainability. Earthscan, Routledge.

Ostrom E., 2007. A diagnostic approach for going beyond panaceas. Science, 104/39, 1518115187.

Ostrom E., 2009. A General Framework for Analyzing Sustainability of Social Ecological Systems. Science 325, 419-422.

Ostrom E., 1990. Governing the Commons. The Evolution of Institutions for Collective Action. Cambridge University Press, Cambridge.

Pecqueur B., 2001. Qualité et développement territorial : l'hypothèse du panier de biens et de services territorialisés. Économie rurale 261, 37-49.

Pettigrew A. M., 1997 What Is a Processual Analysis? Scand. J. Mgmt 13/4, 337-348.

Poteete, A., Janssen, M., Ostrom, E., 2010. Working together. Collective action, the Commons and Multiple Methods in Practice. Princeton University Press, Princeton and Oxford.

Rallet A., Torre A., 2004. Proximité et localisation. Economie Rurale 280, 25-41.

Rambonilaza T., Amblard L., Revol H., 2013. De l'offre paysagère a la production paysagère

: l'économie des chemins ruraux, in : Luginbühl Y., Terrasson D. (coord.) Paysage et Développement Durable. Éditions Quæ, Versailles, 77-87. 
Rey-Valette H., Chia E., Mathé S., Michel L., Nougarèdes B., Soulard C.T., Maurel P., Jarrige F., Barbe E., Guiheneuf P-Y., 2014. Comment analyser la gouvernance territoriale? Mise à l'épreuve d'une grille de lecture. Géographie, Economie, Société 16/1, 65-89.

Rui, S., 1999. L'impasse de l'amont. Débat public et conflits d'aménagement. Annales des ponts et chaussées 92, 47-52.

Schermer M., Renting H., Oostindie H., 2011. Collective farmers' marketing initiatives in Europe: diversity, contextuality and dynamics. International Journal of Sociology of Agriculture and Food 18, 1-11.

Schlager E., Ostrom E., 1992. Property rights regimes and natural resources: a conceptual analysis. Land Economics 68/3, 249-62.

Schmid A.F., Hatchuel A., 2014. On generic epistemology. Angelaki, Journal of the Theoretical Humanities 19/2, 131-144.

Schusler T.M., Decker D.J., Pfeffer M.J., 2003. Social Learning for Collaborative Natural Resource Management. Society and Natural Resources 15, 309-326.

Soulard C., Compagnone C., Lemery B., 2007. La recherche en partenariat : entre fiction et friction. Natures sciences sociétés 15/1, 13-22.

Stengers I., 2006. La Vierge et le Neutrino : Les scientifiques dans la tourmente. Les Empêcheurs de penser en rond/ Seuil, Paris.

Thiel A., Schleyer C., Plieninger T., 2012. Wolves are mobile, while fruit trees are not! How characteristics of resources and supranational regulatory frameworks shape the provision of biodiversity and ecosystem in Germany. Environmental Policy and Governance 22/3, 189204. 
Tilly C., 1985. Models and realities of popular collective action. Social Research 52/4, $717-$ 747.

Torre A., 2015. Théorie du développement territorial. Géographie, économie, société 17/3, 273-288.

Trognon L., 2013. Accompagner la réflexivité, une construction de l'expérience. Education Permanente 196, 65-74.

Truffer B., Störmer E., Maurer M., Ruef, A., 2010. Local Strategic Planning Processes and Sustainability Transitions in Infrastructure Sectors. Environmental Policy and Governance 20, 258-269.

Vinck D., 2009. De l'objet intermédiaire à l'objet-frontière. Revue d'anthropologie des connaissances $3 / 1,51-72$.

Wade R., 1987. The management of common property resources: collective action as an alternative to privatisation or state regulation. Cambridge Journal of Economics 11, 95-106.

Watts D.C.H., Ilbery B., Maye D., 2005. Making Reconnections in Agro-Food Geography: Alternative Systems of Food Provision. Progress in Human Geography 29/1, 22-40. 